Ger J Exerc Sport Res 2022 · 52:468-471 https://doi.org/10.1007/s12662-021-00785-9 Received: 28 October 2021

Accepted: 4 November 2021

Published online: 26 November 2021

(c) The Author(s), under exclusive licence to Springer-Verlag GmbH Deutschland and Bundesinstitut für Sportwissenschaft, Deutscher Olympischer Sportbund, Deutsche Vereinigung für Sportwissenschaft 2021

\author{
Andrew Sortwell ${ }^{1}(\mathbb{D}) \cdot$ Rodrigo Ramirez-Campillo ${ }^{2,3}$ (D) Jorge Knijnik ${ }^{4}(\mathbb{D}) \cdot$ \\ Pedro Forte $^{1,5}$ (D) - Daniel Marinho ${ }^{1,6}$ (D) $\cdot$ Ricardo Ferraz $^{1,6}$ (D) $\cdot$ Kevin Trimble $^{7}$ (D \\ ${ }^{1}$ Research Centre in Sports Sciences, Health Sciences and Human Development (CIDESD), Vila Real, \\ Portugal \\ ${ }^{2}$ Department of Physical Activity Sciences, Universidad de Los Lagos, Santiago, Chile \\ ${ }^{3}$ School of Physical Therapy, Faculty of Rehabilitation Sciences, Universidad Andres Bello, Santiago, Chile \\ ${ }^{4}$ Centre for Educational Research, Western Sydney University, Penrith, Australia \\ ${ }^{5}$ Douro Higher Institute of Educational Sciences, Penafiel, Portugal \\ ${ }^{6}$ Department of Sport Sciences, University of Beira Interior, Covilhã, Portugal \\ ${ }^{7}$ Faculty of Education and Arts, Australian Catholic University, Sydney, Australia
}

\title{
Commentary: Face masks in physical education classes during the COVID-19 delta variant wave: a call for awareness
}

2020). However, the World Health Organisation's recommendations related to physical education (PE) is that special considerations may be required if face masks significantly hinder the learning process and negatively impact the delivery of the curriculum (Krishnaratne et al., 2020).

Even though there is a strengthening of broad recommendations being disseminated by health experts to the broader community, there is a lack of evidence-based guidelines for schools to deliver PE lessons. This paper explores the potential effect of students wearing masks in PE to propose key considerations for using face masks in those lessons during the COVID-19 delta wave. This commentary begins with a description of the central purpose of wearing masks during the COVID19 pandemic. It will also discuss the transmission of COVID-19 to reinforce the purpose of wearing masks. Next, the effect of face masks on movement potential will be explored, followed by an awareness of potential implications for student wellbeing in PE classes. The paper ends with a short discussion on considerations for PE teachers in the uncertain pandemic environment.

\section{Central purpose of the masks}

The health and wellbeing basis for using face masks in the context of the COVID-19 delta wave of the pandemic has been continuously revised and updated. Research published in 2021 suggests the main modes of coronavirus delta variant transmission occurs when an infected person ejects virus particles from their respiratory tract, which an exposed person can absorb through the mouth, nose, or eyes (Hui et al., 2020). Furthermore, growing research suggests transmission of dispersed solid particles or liquid droplets can travel up to $9 \mathrm{~m}$ (Hui et al., 2020; Li et al., 2020) and could potentially be suspended in the air temporarily (Chiu et al., 2020), especially indoors where there can be little air turbulence. One of the factors influencing the distance travelled and suspension in the air is the droplet size ( $\mathrm{Li}$ et al., 2021). Tiny droplets ejected from an infected person are more likely to evaporate faster than larger droplets, and both will evaporate outdoors faster than in an indoor environment (Li et al., 2021). Face masks can provide protection against the droplets ejected from an infected person with the highly contagious coronavirus delta variant, although additional preventative 
strategies such as regular testing, proactive hygiene practices, and social distancing may also be required (Chiu et al., 2020). The level of protection provided by face masks can vary across the different types available, i.e. from cloth style coverings and surgical masks to filtering facepiece level 2 respirators (O'Kelly, Arora, Pirog, Ward, \& Clarkson, 2021). Moreover, the level of protection appears to be relatively stable and independent of sustained activity or duration of wear (Asadi et al., 2020).

Some countries may obligate the use of face masks in outdoor activities, even though there appears to be a reduced risk for the spread of the coronavirus delta variant (Bulfone, Malekinejad, Rutherford, \& Razani, 2021). However, in the outdoor environment, face masks can still reduce the movement of aerosol particles spreading caused by aerodynamic effects of motion, hence reducing the risk of transmission and increasing student safety (Blocken, Malizia, Van Druenen, \& Marchal, 2020). Moreover, for physical activities such as walking, bicycling, hiking, and jogging, at least $5 \mathrm{~m}$ of social distancing has been recommended (Nyenhuis, Greiwe, Zeiger, Nanda, \& Cooke, 2020). Even though face masks are regarded as effective in reducing transmission, social distancing during $\mathrm{PE}$ in combination with face masks may improve the level of protection from coronavirus delta variant. The final decision to use masks in outdoor or indoor PE classes needs to be determined by the local health authorities and government policies, which take into account infection levels, public health status and the latest evidence for reducing transmission.

\section{Effect of face masks on movement potential}

There is limited research examining the impact of face masks on participation, health and wellbeing in PE lessons. The central purpose of face masks is to minimise airflow resulting in a reduced likelihood of viral transmission. For PE students engaged in movement activities, the reduced airflow has the potential to reduce the capacity of students to exert themselves. This reduced airflow may influence high-intensity movement activities.

One study by Tornero-Aguilera, Rubio-Zarapuz, Bustamante-Sánchez, and Clemente-Suárez (2021) examined the effect of surgical mask use on trained runners' performance and physiological measures. The findings showed that wearing the surgical mask significantly reduced performance in a $400 \mathrm{~m}$ running test and blood oxygen saturation, leading to increased perceived exertion and stress. These results are consistent with another study on the athletic population using a graded treadmill test (Driver et al., 2021). Driver et al. (2021) suggest that the use of surgical masks increases the likelihood of lower blood oxygen saturation and raised values of perceived exertion due to cloths masks reducing maximal oxygen consumption by $29 \%$. In studies during resistance training movement activities, face masks can hinder sufficient exertion, preventing study participants from attaining similar metabolic responses as when not wearing a mask (Andre et al., 2018; Motoyama, Joel, Pereira, Esteves, \& Azevedo, 2016; Ramos-Campo et al., 2021).

Exploratory investigations to date have shown face masks to reduce cardiorespiratory capacity and, more specifically, ventilation in high-intensity incremental exercise (Fikenzer et al., 2020). However, face masks have little effect in low to moderate intensity movement activities, as the physiological demands are lower. For example, Roberge, Kim, and Benson (2012) showed that 20 healthy young adults using face masks during one hour of low to moderate-intensity physical activity do not significantly affect performance or perceived exertion. Similarly, a recent multiple crossover trial study for healthy volunteers found that one hour of low-moderate intensity exercise with a surgical mask had no effect on heart rate and blood pressure even though the subjects experienced a slight decrease in arterial oxygen content. In the study, conducted by Fikenzer et al. (2020), 12 healthy adult males were examined for the effect of surgical masks and FFP2/N95 masks on cardiopulmonary exercise capacity. In the randomised cross-over study, both masks had a marked negative impact on exercise parameters such as maximum power output and oxygen uptake, and furthermore, the masks were perceived by participants as impacting breathing resistance. Similarly, in another randomised cross-over study investigating the effects of medical face masks on performance in well-trained athletes, Egger et al. (2021) found that wearing a face mask was also associated with a significant reduction in maximum power output, but not associated with a change in submaximal performance during a stepwise incremental exercise test to exhaustion.

Based on explored research, surgical face and cloth face masks may have little or no negative impact on physiological demands of low to moderate intensity movement activities. It appears that oxygen saturation may decrease during low to moderate movement activities, with no negative impact on health and wellbeing. However, high-intensity movement activities may be affected by the use of face masks. If government health orders or school policies direct teachers to enforce mask-wearing during PE classes, exercises performed at moderate and lower intensity may be better suited under such enforced circumstances.

\section{Wearing face masks in PE-awareness of potential health issues}

There is limited research on the impact of face masks and types of masks (i.e. cloth or surgical) on students in PE. The impact of face mask usage on the teaching and learning in PE could be an impediment, especially where students mobilise both cognitive and physiological capabilities to effectively engage in the lessons, which may compromise student learning and their health and wellbeing. Currently, there is little published data on the potential adverse effects of wearing face masks in PE or physical activity. However, education authorities and schools need to be proactive in supporting student wellbeing; hence risk assessment of students wearing face masks in PE lessons needs to be considered. Students with underlying respiratory conditions such 
as chronic asthma should have a medical assessment to assess the impact of wearing a mask during PE lessons and, if required, receive an exemption from wearing a mask. Based on anecdotal reports (Davison, 2020), students health in PE may be at risk due to higher levels of carbon dioxide which is breathed in (hypercapnia) and a partial decrease in oxygen leading to hypotension (hypoxemia). Potential side effects from mask-wearing warrant further investigation into the likelihood of hypercapnia and hypoxemia whilst engaging in physical activity (Shein et al., 2021).

During PE lessons, the intensity level can vary and require varying demands on the cardiovascular-respiratory system to supply the exercising muscles with blood, oxygen and remove waste products. After the cessation of PE lessons, students will tend to move to their next subject lesson. Most often, the cardiovascular-respiratory system will return to resting levels after PE before commencing the next lesson; however, the system may continue to be activated for a substantial amount of time. In PE, if students are engaged continuously in a four-sided soccer game for $50 \mathrm{~min}$ at a high intensity (near maximal effort), heart rate is more likely to remain slightly elevated compared to normal for a more extended time relative to low intensity and shorter duration (Romero, Minson, \& Halliwill, 2017). After PE, depending on the duration and intensity of the lesson activities, students might be at an increased risk of post-exercise syncope, which is exhibited by slight cognitive confusion and impaired vision (Smith \& Ainslie, 2017). Roberge and colleagues (Roberge et al., 2010) showed that a face mask could slightly increase inhalation and exhalation resistance, resulting in a slight increase in the activity of the respiratory muscles. Therefore, we reinforce the need for an active cool-down at the end of the PE lessons to support the recovery of the physiological systems to accelerate the return to normal before students might be required to wear their mask going to the next lesson.

\section{Recommendations}

Government health orders and education authority guidelines concerning students wearing masks on school premises should be followed. If wearing of masks by students in PE lessons is mandated, we recommend the following pedagogical modifications:

- Lesson intentions and success criteria should be broad and flexible

- Use of small group activities with reduced movement space

- Modified rules which reduce the intensity of the movement activities

- Students actively and frequently collaborate with the teacher in shaping the educational experiences which maintain student wellbeing

- Increased access to regular rest breaks

- Inclusive activities involving students unable to wear a mask due to medical conditions

- Active cool-down at the end of the lesson

Such recommendations mentioned above complement the general guidelines recommended to avoid virus spreading, such as regular hand washing and social distancing.

\section{Conclusion}

The current COVID-19 pandemic is continuously changing with new variants such as the delta and the mu variant of COVID-19 (Uriu et al., 2021). Education authorities and teachers need to be agile and able to adapt to the changing pandemic environment. Based on the limited evidence to date, the recommendations are suggested in conjunction with the authors' extensive paediatric exercise science experience. The latest health orders and recommendations on the use of face masks by government authorities need to be followed to ensure the safety and wellbeing of students. In summary, face masks offer a degree of protection from transmission and may impact the intensity of PE movement activities.

\section{Corresponding address}

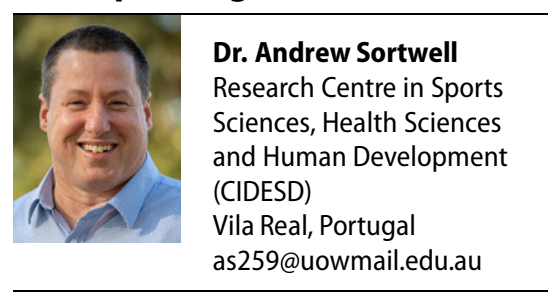

Funding. No sources of funding were used to assist in the preparation of this paper.

\section{Declarations}

Conflict of interest. A. Sortwell, R. Ramirez-Campillo, J. Knijnik, P. Forte, D. Marinho, R. Ferraz and K. Trimble declare that they have no competing interests.

No human subjects were involved, so no ethical approval or data protection was applied for.

\section{References}

Andre, T.L., Gann, J.J., Hwang, P.S., Ziperman, E., Magnussen, M.J., \& Willoughby, D.S. (2018). Restrictivebreathing maskreduces repetitions to failure during a session of lower-body resistance exercise. The Journal of Strength \& Conditioning Research, 32(8), 2103-2108. https://doi.org/10. 1519/jsc.0000000000002648.

Asadi, S., Cappa, C. D., Barreda, S., Wexler, A. S., Bouvier, N.M., \& Ristenpart, W.D. (2020). Efficacy of masks and face coverings in controlling outward aerosol particle emission from expiratory activities. Scientific Reports, 10(1), 15665. https:// doi.org/10.1038/s41598-020-72798-7.

Blocken, B., Malizia, F., Van Druenen, T., \& Marchal, T. (2020). Towards aerodynamically equivalent COVID19 $1.5 \mathrm{~m}$ social distancing for walking and running. Preprint. https://www.euroga.org/ system/1/user_files/files/000/045/111/45111/ 150d3060c/original/Social_Distancing_v20_ White_Paper.pdf.

Bulfone, T.C., Malekinejad, M., Rutherford, G. W., \& Razani, N. (2021). Outdoor transmission of SARSCoV-2 and other respiratory viruses: a systematic review. J Infect Dis, 223(4), 550-561. https://doi. org/10.1093/infdis/jiaa742.

Cha, S. (2021). Wearing masks, South Korean students to go back to school. https://www. reuters.com/article/us-health-coronavirussouthkorea-school-idUSKBN22G0TJ. Last access: 10 September 2021.

Chiu, N., Chi, H., Tai, Y., Peng, C., Tseng, C., Chen, C., Tan, B. F., \& Lin, C. (2020). Impact of wearing masks, hand hygiene, and social distancing on influenza, enterovirus, and all-cause pneumonia during the Coronavirus pandemic: retrospective national epidemiological surveillance study. Journal of medical Internet research,22(8), e21257-e21257. https://doi.org/10.2196/21257.

Davison, K. (2020). Two schoolboys collapse and die just six days apart in China while wearing face masks. https://7news.com.au/lifestyle/ health-wellbeing/two-schoolboys-collapseand-die-just-six-days-apart-in-china-while- 
wearing-face-masks-c-1017871. Lastaccess: 14 September 2021.

Driver, S., Reynolds, M., Brown, K., Vingren, J.L., Hill, D.W., Bennett, M., Gilliland, T., McShan, E., Callender, L., Reynolds, E., Borunda, N., Mosolf, J., Cates, C., \& Jones, A. (2021). Effects of wearing a cloth face mask on performance, physiological and perceptual responses during a graded treadmill running exercise test. British Journal of Sports Medicine. https://doi.org/10. 1136/bjsports-2020-103758.

Egger, F., Blumenauer, D., Fischer, P., Venhorst, A., Kulenthiran, S., Bewarder, Y., Zimmer, A., Böhm, M., Meyer, T., \& Mahfoud, F. (2021). Effects of face masks on performance and cardiorespiratory response in well-trained athletes. Clinical research in cardiology. https://doi.org/10.1007/ s00392-021-01877-0.

England, P.H. (2021). Schools COVID-19 operational guidance. https://www.gov.uk/ government/publications/actions-for-schoolsduring-the-coronavirus-outbreak/schoolscovid-19-operational-guidance. Last access: 30 August 2021.

Fikenzer, S., Uhe, T., Lavall, D., Rudolph, U., Falz, R., Busse, M., Hepp, P., \& Laufs, U. (2020). Effects of surgical and FFP2/N95 face masks on cardiopulmonary exercise capacity. Clinical Research in Cardiology. https://doi.org/10.1007/s00392-020-01704-y.

Guell, O. (2021). Coronavirus restrictions to remain in Spanish schools, with half of high school students due to be vaccinated. https://english. elpais.com/society/2021-08-23/coronavirusrestrictions-to-remain-in-spanish-schoolswith-half-of-high-school-students-due-tobe-vaccinated.html. Last access: 29 August 2021.

Hui, K.P.Y., Cheung, M. C., Perera, R. A.P.M., Ng, K.C., Bui, C.H.T., Ho, J.C.W., Ng, M.M.T., Kuok, D.I.T., Shih, K.C., Tsao, S.W., Poon, L.L.M., Peiris, M., Nicholls, J.M., \& Chan, M.C.W. (2020). Tropism, replication competence, and innate immune responses of the coronavirus SARS-CoV-2 in human respiratory tract and conjunctiva: an analysis in ex-vivo and invitro cultures. The Lancet Respiratory Medicine, 8(7), 687-695. https://doi.org/10.1016/S22132600(20)30193-4.

Krishnaratne, S., Pfadenhauer, L. M., Coenen, M., Geffert, K., Jung-Sievers, C., Klinger, C., et al. (2020). Measures implemented in the school setting to contain the COVID-19 pandemic: a rapid scoping review. Cochrane Database of Systematic Reviews. https://doi.org/10.1002/ 14651858.CD013812.

Lechien, J.R., \& Saussez, S. (2021). Importance of epidemiological factors in the evaluation of transmissibility and clinical severity of SARS-CoV-2 variants. The Lancet Infectious Diseases. $\quad$ https://doi.org/10.1016/S14733099(21)00474-6.

Li, H., Leong, F.Y., Xu, G., Kang, C.W., Lim, K.H., Tan, B. H., \& Loo, C. M. (2021). Airborne dispersion of droplets during coughing: a physical model of viral transmission. Scientific Reports, 11(1), 4617. https://doi.org/10.1038/s41598-021-84245-2.

Motoyama, Y. L., Joel, G. B., Pereira, P. E. A., Esteves, G. J., \& Azevedo, P.H.S.M. (2016). Airflow-restricting mask reduces acute performance in resistance exercise. Sports, 4(4), 46.

Nyenhuis, S. M., Greiwe, J., Zeiger, J.S., Nanda, A., \& Cooke, A. (2020). Exercise and fitness in the age of social distancing during the COVID-19 pandemic. The journal of allergy and clinical immunology. In practice, 8(7), 2152-2155. https://doi.org/10. 1016/j.jaip.2020.04.039.

O'Kelly, E., Arora, A., Pirog, S., Ward, J., \& Clarkson, P. J. (2021). Comparing the fit of N95, KN95, surgical, and cloth face masks and assessing the accuracy of fit checking. PLoS One, 16(1), e245688. https:// doi.org/10.1371/journal.pone.0245688.

Ramos-Campo, D. J., Pérez-Piñero, S., Muñoz-Carrillo, J.C., López-Román, F.J., García-Sánchez, E., \& Ávila-Gandía, V. (2021). Acute effects of surgical and FFP2 face masks on physiological responses and strength performance in persons with Sarcopenia. Biology, 10(3), 213.

Roberge, R. J., Bayer, E., Powell, J. B., Coca, A., Roberge, M. R., \& Benson, S. M. (2010). Effect of exhaled moisture on breathing resistance of $\mathrm{N} 95$ filtering facepiece respirators. Ann Occup Hyg, 54(6), 671-677. https://doi.org/10.1093/annhyg/ meq042.

Roberge, R. J., Kim, J. H., \&Benson, S. M. (2012). Absence of consequential changes in physiological, thermal and subjective responses from wearing a surgical mask. Respir Physiol Neurobiol, 181(1), 29-35. https://doi.org/10.1016/j.resp.2012.01. 010.

Romero, S. A., Minson, C. T., \& Halliwill, J.R. (2017). The cardiovascular system after exercise. J App/ Physiol, 122(4), 925-932. https://doi.org/10. 1152/japplphysiol.00802.2016.

Shein, S. L., Whitticar, S., Mascho, K. K., Pace, E., Speicher, R., \& Deakins, K. (2021). The effects of wearing facemasks on oxygenation and ventilation at rest and during physical activity. PLoS One, 16(2), e247414-e247414. https://doi.org/10.1371/ journal.pone.0247414.

Smith, K. J., \& Ainslie, P. N. (2017). Regulation of cerebral blood flow and metabolism during exercise. Experimental Physiology, 102(11), 1356-1371. https://doi.org/10.1113/EP086249.

Spitzer, M. (2020). Masked education? The benefits and burdens of wearing face masks in schools during the current Corona pandemic. Trends in neuroscience and education, 20,100138-100138. https://doi.org/10.1016/j.tine.2020.100138.

Tornero-Aguilera, J. F., Rubio-Zarapuz, A., BustamanteSánchez, A., \& Clemente-Suárez, V. J. (2021). The effect of surgical mask use in anaerobic running performance. Applied Sciences, 11(14), 6555. https://doi.org/10.3390/app11146555.

Uriu, K., Kimura, I., Shirakawa, K., Takaori-Kondo, A., Nakada, T.A., Kaneda, A., Consortium, T.G.T.P.J., Nakagawa, S., \& Sato, K. (2021). Ineffective neutralization of the SARS-CoV-2 Mu variant by convalescent and vaccine sera. bioRxiv, 2021.2009.2006.459005. https://doi.org/10. $1101 / 2021.09 .06 .459005$. 